\title{
Polypeptide and Peptide Neurotoxins for Identification of Nicotinic Acetylcholine Receptors and for Study of Their Structure
}

\author{
I. E. Kasheverov, Yu. N. Utkin, I. V. Shelukhina, E. V. Kryukova, D. A. Kuzmin, A. V. Osipov, \\ M. N. Zhmak, and V. I. Tsetlin \\ Shemyakin-Ovchinnikov Institute of Bioorganic Chemistry, Russian Academy of Sciences, Moscow; \\ e-mail:iekash@mx.ibch.ru
}

DOI: $10.1134 / \mathrm{S} 1990747809030258$

There are two types of nicotinic acetylcholine receptors (nAChRs): neuronal and muscle type. Muscle-type nAChRs consist of two $\alpha$-subunits $(\alpha 1)$ as well as one $\beta$-, $\gamma$ - (or $\varepsilon$ - in mature form) and $\delta$-subunit each. The subunits form pentameric complex, along the central axis of which an ionic channel spanning the membrane is located. The structural information about muscle-type nAChR was obtained mainly by the study of the receptor from the electric organ of Torpedo. Pentameric neuronal nAChRs consist of only two types of subunits: $\alpha(\alpha 2-\alpha 10)$ and $\beta(\beta 2-\beta 4)$. Neuronal $n A C h R s$ can exist either as heteromers (i.e., consisting of both $\alpha$ - and $\beta$-subunits) or homomers formed by $\alpha$-subunits only (the most studied are $\alpha 7 \mathrm{nAChRs).}$ Neuronal nAChRs are involved in cognition, pain signal transduction, and release of different neurotransmitters. During the last decade, neuronal nAChRs were found in cells of skin and lung as well as in the immune system, where they take part in regulation of inflammation and growth. The aim of fundamental studies on $n A C h R s$ is to ascertain the details of their spatial structure as well as to establish the mechanisms coupling the ligand binding with opening of ion channel and subsequent steps of signal transduction. Information about ligand-binding sites serves as a basis for the design of new generation drugs. Application directed task is to develop the method for identification of different nAChR subtypes in tissues, as malfunctioning of these receptors results in different muscle dystrophies, mental and neurodegenerative diseases. Peptide and polypeptide neurotoxins play an important role in fulfilment of both tasks. Thirty years ago $\alpha$-neurotoxins allowed purifying Torpedo nAChR and in 2001 they helped to identify a fresh-water mollusc acetylcholinebinding protein (AChBP), which is a perfect structurefunctional model of nAChR ligand-binding domain. $\alpha$-Conotoxins, neurotoxic peptides from poisonous sea molluscs of Conus genus, selectively block different nAChR subtypes. Here we present the results of the studies on Torpedo and $\alpha 7 \mathrm{nAChRs}$ as well as AChBP carried out in our laboratory in collaboration with colleagues abroad. The established X-ray structure of $\mathrm{AChBP}$ with bound $\alpha$-conotoxin showed that the principal differences in binding agonists and antagonists are manifested in position of $\mathrm{AChBP}$ loop $\mathrm{C}$ containing vicinal disulphide. The computational models of different nAChR subtypes were constructed. Using these models, new $\alpha$-conotoxin analogues were designed by means of docking and molecular dynamics and synthesized by solid phase peptide synthesis. Synthetic analogue of $\alpha$-conotoxin SIA possessing 200 times higher affinity to Torpedo nAChR as compared to natural SIA was prepared. The binding sites of photoactivatable $\alpha$-conotoxin GI analogue on Torpedo nAChR were characterized. $\alpha$-Cobratoxin dimer, in which $\alpha$-cobratoxin monomers are bound by disulfide bridges, was isolated from cobra Naja kaouthia venom. Similarly to $\alpha$-cobratoxin, this dimer is capable to bind to AChBP, Torpedo and $\alpha 7$ nAChRs; moreover, it acquires the capacity to bind to $\alpha 3 \beta 2 \mathrm{nAChR}$. Cell line GH4C1 stably expressing $\alpha 7 \mathrm{nAChR}$ was used to analyze the interaction of fluorescently labelled derivatives of $\alpha$-cobratoxin and $\alpha$-bungarotoxin with receptor. It was shown that specificity of interaction can be proved by use of $\alpha$-cobratoxin and short-chain neurotoxin in competition experiments. These two toxins and fluorescently labelled $\alpha$-bungarotoxin were applied to detect $\alpha 7 \mathrm{nAChR}$ on tissue sections.

This work was supported by grants from RFBR (project nos. 08-04-00801, 06-04-49198, and 06-0448982), Program of the Russian Academy of Sciences Molecular and Cell Biology, and FP7 (Neurocypres) of EC. 